\title{
Epilepsy outcome following resection of low-grade brain tumors in children
}

\author{
Arvind C. Mohan, MD, ${ }^{1}$ Howard L. Weiner, MD, ${ }^{1}$ Carrie A. Mohila, MD, PhD, ${ }^{2}$ \\ Adekunle Adesina, MD, PhD, ${ }^{2}$ Murali Chintagumpala, MD, ${ }^{3}$ Daniel Curry, MD, ${ }^{1}$ Andrew Jea, MD, ${ }^{4}$ \\ Jonathan J. Lee, MD, ${ }^{5}$ Sandi K. Lam, MD, MBA, ${ }^{1}$ William E. Whitehead, MD, ${ }^{1}$ Robert Dauser, MD, ${ }^{1}$ \\ Daniel Yoshor, MD, ${ }^{1}$ and Guillermo Aldave, MD, PhD ${ }^{1}$
}

\begin{abstract}
1Division of Pediatric Neurosurgery, Department of Surgery, Texas Children's Hospital, and Department of Neurosurgery, Baylor College of Medicine; ' $D$ epartment of Pathology, Texas Children's Hospital, Baylor College of Medicine; and ${ }^{3}$ Cancer and Hematology Center, Texas Children's Hospital, Baylor College of Medicine, Houston, Texas; ${ }^{4}$ Section of Pediatric Neurosurgery, Riley Hospital for Children, Department of Neurological Surgery, Indiana University School of Medicine and Goodman Campbell Brain and Spine, Indianapolis, Indiana; and 5Department of Neurosurgery, Houston Methodist Hospital, Houston, Texas
\end{abstract}

OBJECTIVE The indication for and timing of surgery for epilepsy associated with low-grade mixed neuronal-glial tumors may be controversial. The purpose of this study was to evaluate the effect of resection and associated variables on epilepsy and on progression-free survival (PFS).

METHODS A retrospective chart review of patients treated between 1992 and 2016 was conducted to identify individuals with epilepsy and low-grade gliomas or neuronal-glial tumors who underwent resective surgery. Data analyzed included age at epilepsy onset, age at surgery, extent of resection, use of electrocorticography, the number of antiepileptic drugs (AEDs) before and after surgery, the presence of dysplasia, Engel class, histological findings, and PFS. The institutional review board protocol was specifically approved to conduct this study.

RESULTS A total of 107 patients were identified. The median follow-up was 4.9 years. The most common pathology was dysembryoplastic neuroepithelial tumor (36.4\%), followed by ganglioglioma (31.8\%). Eighty-four percent of patients had Engel class I outcomes following surgery. Gross-total resection was associated with a higher likelihood of an Engel class I outcome $(90 \%)$ as compared to subtotal resection $(58 \%)(p=0.0005)$. Surgery reduced the AED burden, with $40 \%$ of patients requiring no AEDs after surgery $(p<0.0001)$. Children with neurodevelopmental comorbidities $(n=5)$ uniformly did not experience seizure improvement following resection $(0 \%$ vs $83 \%$ overall; $p<0.0001)$. Electrocorticography was used in $33 \%$ of cases and did not significantly increase class I outcomes. PFS was $90 \%$ at 5 years. Eleven percent of tumors recurred, with subtotal resection more likely to result in recurrence (hazard ratio 5.3, $p=0.02$ ). Histological subtype showed no significant impact on recurrence.

CONCLUSIONS Gross-total resection was strongly associated with Engel class I outcome and longer PFS. Further studies are needed to elucidate the suitable time for surgery and to identify factors associated with oncological transformation.

https://thejns.org/doi/abs/10.3171/2019.1.PEDS18367

KEYWORDS epilepsy; low-grade brain tumors; outcome; oncology

$\mathrm{L}$ OW-GRADE, epilepsy-associated, neuronal-glial tumors are an important subset of tumors that cause epilepsy, typically with early-childhood onset. These tumors are only $2 \%-5 \%$ of brain tumors but are the second most common lesion associated with seizures in those patients admitted for epilepsy surgery. ${ }^{2,4}$ Stud- ies suggest that they may be responsible for up to $95 \%$ of tumor-related epilepsy cases. The advent of molecular genotyping has permitted further characterization of these seemingly heterogeneous tumors into a unified group. ${ }^{2}$ As such, management techniques for these tumors will continue to evolve.

ABBREVIATIONS AED = antiepileptic drug; ECoG = electrocorticography; GTR = gross-total resection; HR = hazard ratio; PFS = progression-free survival; STR = subtotal resection.

SUBMITTED June 11, 2018. ACCEPTED January 14, 2019.

INCLUDE WHEN CITING Published online March 22, 2019; DOI: 10.3171/2019.1.PEDS18367. 
There are no definitive guidelines on the management of these tumors. Even in existing guidelines, the role of antiepileptic drugs (AEDs) in treating epilepsy is not fully understood. ${ }^{21}$ Existing data are particularly poor for recommendations on management in pediatric patients ${ }^{19}$ and reveal that typically, patients do not undergo surgery until adulthood, despite receiving a diagnosis in childhood. ${ }^{1}$ However, there is strong evidence that surgery, particularly when performed early, has a high chance of resulting in an Engel class I outcome. ${ }^{5,7,13,18}$

There is debate about what factors predict the best epilepsy outcome after surgery. Generally, most studies report lesionectomy as the optimal surgical treatment. ${ }^{7}$ However, given the literature suggesting that the seizure focus may extend beyond the lesion, questions remain about the optimal strategy. The role of the total resection and electrocorticography $(\mathrm{ECoG})$ in predicting Engel class I outcomes is still debated., ${ }^{1,2}$

Last, data exist to support the idea that oncological follow-up and treatment is essential for these tumors. ${ }^{9} \mathrm{~A}$ small percentage of these neuronal-glial tumors can become malignant. One study reports that some tumors recurred as malignancies. ${ }^{11}$ It is therefore important to quantify the features that predict recurrence and malignancy. ${ }^{12}$

The primary aim of this study was to determine epilepsy outcome in pediatric patients who underwent surgery for resection of low-grade neuronal-glial tumors. We sought to determine the optimal time for surgical intervention for these epilepsy-associated tumors and to define what factors correlate with tumor recurrence and progression.

\section{Methods}

A retrospective chart review of patients treated between 1992 and 2016 was conducted to identify patients with epilepsy who underwent resection of an epilepsy-associated low-grade tumor. The database used was Epic. Patients were then further selected based on presence of epilepsy and histological subtype consistent with a low-grade glioma, neuronal lesion, or neuronal-glial tumor. Data analyzed included age at epilepsy onset, age at surgery, extent of resection, the presence of neurodevelopmental comorbidities, use of ECoG, the number of AEDs before and after surgery, the presence of dysplasia, Engel class, tumor histology, and progression-free survival (PFS). All patients had undergone MRI with and without contrast pre- and postoperatively.

Patients must have received a formal diagnosis of epilepsy in order to qualify for inclusion in this study. Therefore epilepsy was defined as at least 2 unprovoked seizures and the patient had to start on AEDs. ${ }^{6}$ Furthermore, of the patients with epilepsy, only those with histological subtypes specifically identified as a low-grade glioma or lowgrade epilepsy-associated neuroepithelial tumor ${ }^{2}$ were included for analysis. Neurodevelopmental comorbidities were defined as any syndrome or structural abnormality in the brain. All patients underwent EEG under direction of the epilepsy neurology service.

Every case was assessed at a multidisciplinary epilepsy conference, with the participation of neurosurgery, neurol- ogy, neurophysiology, and radiology. The clinical details, imaging, and EEG readings were analyzed in detail. The agreed surgical plan was determined for each case, but overall the aim was the maximum safe resection of the predefined epileptogenic zone. This always included the tumor, in addition to other abnormalities seen on presurgical imaging, like dysplasia or mesial temporal sclerosis. Therefore, subtotal resection (STR) was done only when complete removal of the tumor would carry a potential permanent deficit. The indication for ECoG was assessed individually at the multidisciplinary conference. In general, it was used for those cases in which EEG suggested a potential ictal onset beyond the tumor margin, especially in the presence of an underlying abnormality on presurgical imaging.

The pathological diagnosis was determined by the pediatric neuropathology team from our institution based on the current WHO classification at that time.

Extent of resection was assessed by neuroradiology. Gross-total resection (GTR) was mainly defined as the absence of tumor determined on the preoperative MRI on T2-weighted sequences, as well as the absence of contrast on the T1-weighted postgadolinium sequence if the tumor had shown contrast enhancement prior to surgery. STR was defined as the presence of any type of residual tumor.

The study was conducted under an institutional review board protocol approved by the Baylor College of Medicine.

\section{Statistical Analyses}

Univariate analysis was performed initially to identify factors associated with our primary epileptic outcome measurement (Engel class), and our primary oncological outcome measurement (PFS). Logistic regression multivariate analyses were performed for Engel outcome variables and Cox regression analysis was performed for PFS. SPSS was used for multivariate analyses and Prism was used for univariate odds ratios and descriptive statistics.

\section{Results}

A total of 107 patients were identified based on the aforementioned criteria. Demographic data are listed in Table 1. All patients had focal epilepsy, with $18 \%$ of patients $(n=19)$ also experiencing generalized seizures in addition to focal seizures. The average time to surgery was 2.5 years from the diagnosis of epilepsy, with $45 \%$ of patients undergoing surgery within the 1st year (early surgery) versus $55 \%$ of patients beyond the 1st year (late surgery). The mean follow-up after surgical intervention was 4.8 years (range $0-19$ years).

All surgeries were performed by the same pediatric neurosurgery group of 5 neurosurgeons in a single institution. Fifty-seven percent of cases $(n=61)$ underwent only lesionectomy, with the remaining $43 \%(n=46)$ undergoing additional resection in addition to tumor removal (for example, additional cortical resection $[\mathrm{n}=10]$, lobectomy $[\mathrm{n}=25]$, or amygdalohippocampectomy [ $\mathrm{n}=11])$. Twentyone percent $(n=10)$ of these 46 patients presented with a histological subtype of cortical dysplasia. ECoG was used in $33 \%$ of cases $(n=35)$. 
TABLE 1. Histological diagnosis of 107 tumors analyzed in the study

\begin{tabular}{lc}
\hline \multicolumn{1}{c}{ Histology } & No. of Tumors $(\%)$ \\
\hline DNET & $39(36.4)$ \\
\hline Ganglioglioma & $34(31.8)$ \\
\hline Diffuse astrocytoma, grade II & $12(11.2)$ \\
\hline Pilocytic astrocytoma & $3(2.8)$ \\
\hline Angiocentric glioma & $3(2.8)$ \\
\hline Oligodendroglioma & $2(1.9)$ \\
\hline Anaplastic ganglioglioma & $2(1.9)$ \\
\hline Pleomorphic xanthoastrocytoma & $1(0.9)$ \\
\hline Pilomyxoid astrocytoma & $1(0.9)$ \\
\hline Other* & $10(9.3)$ \\
\hline
\end{tabular}

DNET = dysembryoplastic neuroepithelial tumor.

* Includes mixed histology and nonspecified low-grade neuronal-glial tumor.

Regarding the location of the tumor, $72 \%$ of patients had temporal lesions $(n=77)$, with the remainder in the frontal lobe (14\%), parietal lobe (11\%), and occipital lobe (2\%). Regarding AEDs, $54.4 \%$ of patients were on 1, $20 \%$ were on 2 , and the remaining $25.6 \%$ were on 3 or more AEDs.

Pathological analysis showed the following: dysembryoplastic neuroepithelial tumor (36.4\%), ganglioglioma $(31.8 \%)$, diffuse astrocytoma (11.2\%), pilocytic astrocytoma (2.8\%), angiocentric glioma (2.8\%), oligodendroglioma (1.9\%), anaplastic ganglioglioma (1.9\%), pilomyxoid astrocytoma (0.9\%), and pleomorphic xanthoastrocytoma $(0.9 \%)$, with the remaining $9.3 \%$ being mixed histology or nonspecified low-grade neuronal-glial tumor. Table 1 lists these histological subtypes.

Five patients presented with additional neurological comorbidities; all of them presented with severe global developmental delay. Only 1 involved the scenario of a known genetic syndrome (Williams-Beuren, del 7q). The other 4 presented with static encephalopathy due to cerebral palsy $(n=2)$, postinfantile spasm with developmental cortical malformations $(n=1)$, and autism $(n=1)$. None of them had an Engel class I outcome after tumor resection. For those who had EEG records from prior surgery $(\mathrm{n}=$ 4), in $3(75 \%)$ we found spike activity beyond the tumor location (bilateral foci, multiple foci, or $3-\mathrm{Hz}$ generalized spike and wave complexes in the scenario of WilliamsBeuren syndrome). One had a focus of spike over the tumor location, but GTR could not be achieved because the tumor was close to an eloquent area.

In our data set, ECoG was used in 35 cases (33\%). Of these 35 cases, operative details on the use of ECoG were available through operative notes in 22 cases (62.9\%), comprehensive operative notes were not available in 8 cases, and the use of ECoG was not mentioned in the operative reports of 5 cases. ECoG was used prior to resection of the tumor in all 22 cases, and helped define meaningful information in $100 \%$ of cases. In 17 of 22 cases (77.3\%), ECoG helped confirm the presence of epileptogenic activity in the lesion and surrounding brain. In the other 5 cases (22.7\%), ECoG helped confirm the absence of epileptogenic activity in the lesion (i.e., confirmed a silent le- sion). In 6 cases, ECoG was used postresection to confirm preresection epileptogenic activity in tissue that was not resected or to assess epileptogenic activity once the lesion was resected. In 1 case, ECoG helped define the central sulcus by means of phase reversal. Of the 22 cases, the use of ECoG helped change the surgical plan in 7 cases $(31.8 \%)$. In 6 of these cases, the use of ECoG led to the resection of more surrounding tissue than anticipated due to significant epileptogenic activity, and in 1 of these cases, ECoG led to the sparing of tissue due to the absence of significant epileptogenic activity.

\section{Surgery Outcomes}

GTR was achieved in $76 \%$ of patients $(n=81)$. Four patients had complications (3.7\%). Of these, 3 had transient complications (visual field defect, supplementary motor area syndrome, and hemiparesis), and 1 had a permanent deficit due to an anterior cerebral artery stroke.

Ten percent of patients $(n=11)$ required reoperation. Two patients underwent additional tumor resection due to seizure recurrence, and the remainder underwent reoperation because of tumor recurrence $(n=5)$ or progression $(n=4)$.

Among the patients with tumor recurrence $(\mathrm{n}=14)$, none of those who had the recurrence with the same tumor histology or grade $(n=10)$ presented with histological features that could predict an eventual recurrence (e.g., mitosis, vascular proliferation, or anaplastic features). Among the patients with tumor progression to a higher grade at recurrence $(n=4), 50 \%(n=2)$ presented with atypical features like diffusely infiltrative nature, eosinophilic granular bodies, and focally increased MIB-1/Ki-67 up to $14 \%-15 \%$.

\section{Epilepsy Outcomes}

Eighty-four percent of patients $(n=90)$ were seizure free (Engel class I) at long-term follow-up. If we consider only patients with $\geq 3$ and 5 years of follow-up (89 and 69 patients, respectively), the Engel class I rate was $84 \%$ and $81 \%$, respectively. The average number of AEDs was reduced from 1.86 to 0.83 ( $\mathrm{p}<0.0001$ ), and $41 \%$ of patients $(\mathrm{n}=44)$ received no AEDs at all.

Univariate analysis revealed that the factors most significantly associated with Engel class I outcome were GTR as compared to STR (hazard ratio [HR] 6.7, p = 0.0005). Time to surgery, age, sex, tumor histology, use of ECoG, and location (extratemporal vs temporal) had no significant impact on class I outcomes. The presence of other neurodevelopmental comorbidities such as structural abnormalities or other syndromes made the likelihood of class I outcomes significantly less likely (HR 0.014, p $<0.005)$. On multivariate analysis, GTR was significantly associated with an Engel class I outcome (HR 23,315, p $=0.002$ ). Considering for the multivariate analysis only patients with $\geq 3$ years of follow-up, among the variables only GTR and the absence of neurodevelopmental comorbidities presented a significant impact. Table 2 describes the factors associated with an Engel class I outcome in both univariate and multivariate analysis and for patients with $\geq 3$ years of follow-up. 
TABLE 2. Univariate and multivariate analysis of variables associated with seizure-free outcome (Engel class I) following resection

\begin{tabular}{lccc}
\hline \multicolumn{1}{c}{ Group } & Univariate HR (p value) & Multivariate HR ( $p$ value) & Multivariate HR, $\geq 3$ Yrs' FU ( $p$ value) \\
\hline Surgery w/in 1 yr of diagnosis & $3.1(\mathrm{NS})$ & $2.864(\mathrm{NS})$ & $0.99(\mathrm{NS})$ \\
\hline Extent of resection, GTR vs STR & $6.7(0.0005)$ & $23,315(0.002)$ & $5.43(0.02)$ \\
\hline Neurodevelopmental comorbidities & $0.014(<0.005)$ & $1.97(0.0001)$ & $30.52(<0.005)$ \\
\hline Age at surgery & $2.4(\mathrm{NS})$ & $0.057(\mathrm{NS})$ & $0.6(\mathrm{NS})$ \\
\hline Sex & $2.7(\mathrm{NS})$ & $-0.083(\mathrm{NS})$ & $3.1(\mathrm{NS})$ \\
\hline Histology & $1.212(\mathrm{NS})$ & $0.023(\mathrm{NS})$ & $0.06(\mathrm{NS})$ \\
\hline ECoG & $2.7(\mathrm{NS})$ & $-0.045(\mathrm{NS})$ & $1.21(\mathrm{NS})$ \\
\hline Location, ET vs temporal & $1.05(\mathrm{NS})$ & $-0.084(\mathrm{NS})$ & $0.176(\mathrm{NS})$
\end{tabular}

\section{Tumor Outcomes}

The PFS at 5 years was $90 \%$. Four percent of tumors $(n=4)$ recurred with a higher grade, with anaplastic features such as hypercellularity, atypia, and mitosis; and 2 patients had tumors that progressed to glioblastoma. Fifteen percent of patients $(n=16)$ received adjunct radiation therapy. STR was the factor most likely to result in tumor recurrence on univariate analysis (HR 5.3, p = 0.02), and was also significantly associated with recurrence on Cox regression analysis. Histology had no effect on recurrence. Age at surgery, tumor histology, early surgery, extent of resection, and presence of malignant features had no impact on PFS on Cox regression analysis.

Table 3 describes the factors associated with PFS. Figure 1 shows the Kaplan-Meier curve for seizure freedom and PFS.

\section{Discussion}

The purpose of this study was to determine those factors that predict epilepsy outcome after resection of lowgrade brain tumors in pediatric patients. The study included 107 children and is one of the largest series to date in this population. ${ }^{6,9,12}$

Our Engel class I outcome of $84 \%$ was consistent with previously published studies. ${ }^{3,5,8,13,18}$ Interestingly, Englot et al. found a similar rate of Engel class I in the largest systematic literature review of seizure outcomes after resection of neuronal-glial tumors. The review included both adult and pediatric cases, although the results did not differ significantly between these age groups. As in our series, the main prognostic factor for seizure-free outcome was GTR. The complication rate we report (3.7\%) is con-

TABLE 3. Univariate and multivariate analysis of prognostic factors with a potential impact on PFS following resection

\begin{tabular}{llc}
\hline \multicolumn{1}{c}{ Variable } & \multicolumn{1}{c}{$\begin{array}{c}\text { Univariate HR } \\
(\mathrm{p} \text { value })\end{array}$} & $\begin{array}{c}\text { Multivariate HR } \\
(\mathrm{p} \text { value })\end{array}$ \\
\hline Age at surgery & $0.258(\mathrm{NS})$ & $0.022(\mathrm{NS})$ \\
\hline Histology & $1.8(\mathrm{NS})$ & $-0.141(\mathrm{NS})$ \\
\hline Surgery w/in 1 yr of diagnosis & $0.071(\mathrm{NS})$ & $-0.474(\mathrm{NS})$ \\
\hline Extent of resection, GTR vs STR & $5.3(0.02)$ & $1.399(0.045)$ \\
\hline
\end{tabular}

sistent with what Faramand et al. ${ }^{6}$ have published (4.5\%). All surgical complications except one were transient, and all patients went on to have a class I outcome. Therefore, operating on these patients may allow them to have lives free of epilepsy, with minimal additional risk.

Successful epilepsy surgery has several benefits for children. Patients who are maintained on unsuccessful drug regimens show worse recall skills and are at higher risk for cognitive and psychiatric problems. ${ }^{15}$ Even in infants, untreated epilepsy can lead to long-term cognitive decline. ${ }^{20}$ Therefore, in pediatric patients, it becomes uniquely important to identify good candidates for epilepsy surgery and treat them appropriately.

The factor most likely to result in class I outcomes on multivariate analysis was GTR of the tumor. However, early surgery within the 1st year from diagnosis of epilepsy did not have a significant impact on the seizure-free outcome in our study, contrary to the results shown by Englot et al. ${ }^{3}$ Their findings would support an early referral for surgery. Histology, age at diagnosis, sex, and ECoG showed no significant impact on class I outcomes. A particular substratified analysis based on tumoral lineage (neuronal-glial tumors vs low-grade astrocytoma) was performed and found no differences regarding epilepsy or tumor outcomes.

The use of ECoG remains controversial in the treatment of these tumors. Several studies have shown a benefit to $\mathrm{ECoG},{ }^{7,17}$ whereas others, ours included, cannot provide evidence to support its use.22 The purpose of ECoG in these studies is to support full identification of the epileptic zone and, in theory, increase the likelihood of an Engel class I outcome. However, the studies have shown mixed data, with some studies showing no increased likelihood of a class I outcome. ${ }^{22}$ In our study, ECoG was used in 33\% of the patients, and selection bias for the more challenging cases could have muted the effect of the ECoG. Besides, the absence of sufficient power-we only have 35 patients with ECoG-could explain the lack of significance on this analysis. The role of ECoG for tumor-related epilepsy surgery remains unclear, and further studies would be needed to better define its impact.

Patients with neurodevelopmental comorbidities or other structural brain abnormality were unlikely to have a class I outcome following surgery for a low-grade tumor. ${ }^{8}$ 

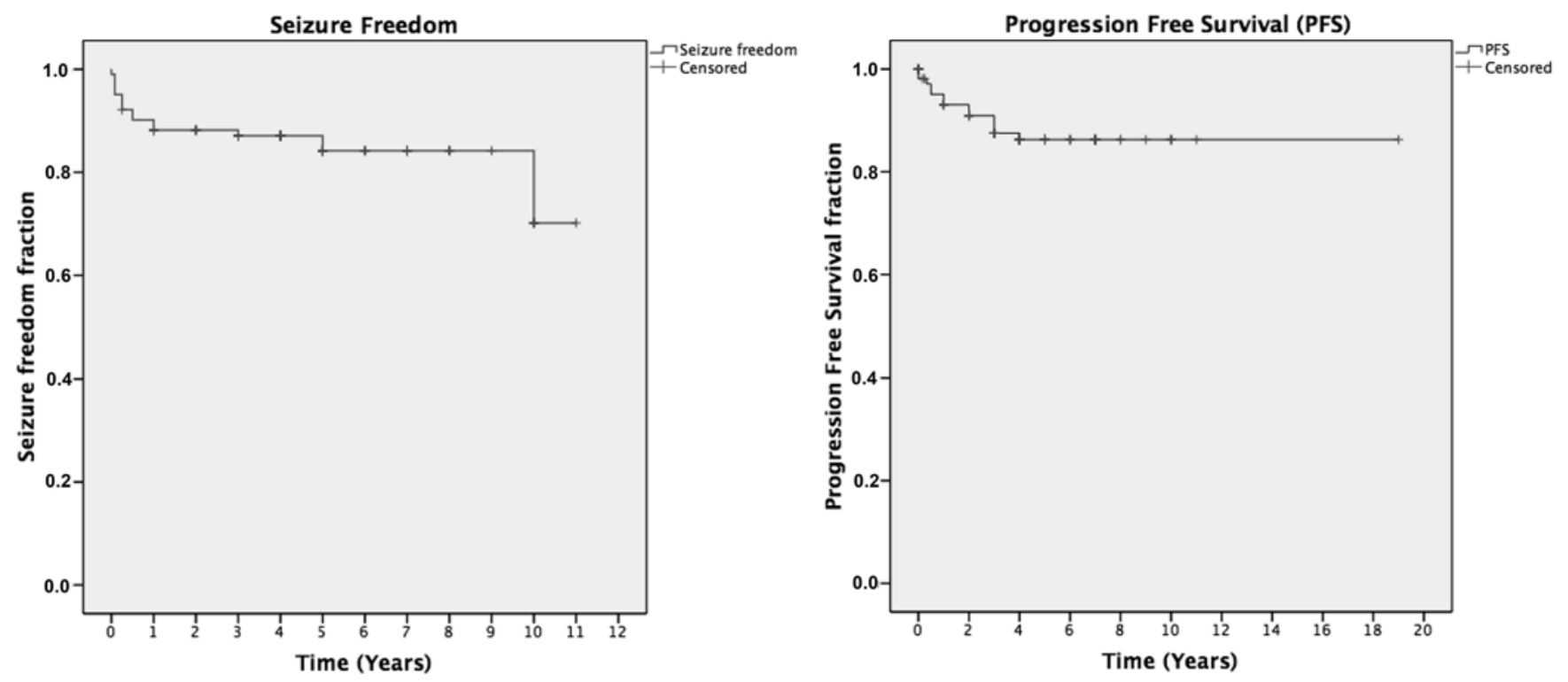

FIG. 1. Kaplan-Meier curves for seizure freedom and PFS.

Patients with lissencephaly, microgyria, or other neuronal migration abnormalities, for example, are at a significantly increased risk for epilepsy. ${ }^{10}$ In our series, most of the patients with neurodevelopmental comorbidities presented, even on the first EEG, with multiple epileptogenic foci beyond the tumor location, and some of them bilaterally. The poor correlation between the EEG findings and tumor location would explain the hesitation to intervene early, as is shown by the time to surgery since diagnosis ( 8 years) in the patients with neurodevelopmental comorbidities versus 2.2 years for the other patients. A long time with intractable seizures since diagnosis plays a key role in the establishment of an epileptic encephalopathy. However, given the initial EEG with multiple epileptogenic foci, we believe that the poor prognosis for an Engel class I outcome in these patients would come mainly from underlying inherent structural anomalies in the brain substance that are present at the beginning, rather than encephalopathy developed after years of intractable seizures. These patients are unlikely to achieve class I outcomes with a lesionectomy alone. The risk/benefit analysis of surgery should be thoroughly assessed individually.

Only STR was associated with recurrence on univariate and multivariate analysis. This would indicate that the extent of resection-particularly a complete resection-is the most important factor associated with both seizurefree outcome and PFS. The rest of the features in the Cox regression were not proved to be significant for malignancy and recurrence. Among the tumor recurrences there were no significant differences in the histology or presence of anaplastic features, but in $50 \%$ of the patients with tumor progression to a higher grade, the lesion had a diffusely infiltrative nature on the initial histology and a mild elevation of the MIB-1/Ki-67 (up to $14 \%-15 \%$ ). Besides, half of the patients who had malignant transformation had a GTR, and 2 of the patients died, which suggests that follow-up with serial imaging should be mandatory regardless of residual tumor.
A major limitation of this study was its retrospective nature and the long period of time over which patients were identified. The retrospective design presents more challenges to accurate assessment from a chart review of important variables like the role of the ECoG or the reason for an STR. Additionally, we were not able to provide information about any cognitive or quality of life outcomes in patients following surgery, because not all patients received follow-up neuropsychological testing. This would have provided stronger support for early surgery in our patients, as evidenced by Faramand et al. ${ }^{6}$ and Ramantani et al..$^{14}$

It is difficult to account for the variability of surgical techniques or technology over this 25 -year period. Most importantly, technical advances in identifying seizure foci in presurgical evaluation have significantly increased the proportion of patients with epilepsy for whom surgery can be an option..$^{16}$ These improvements could have added additional bias in our study. Future studies can further characterize the role of resection of these tumors. Randomized controlled trials may further elucidate the role of ECoG and the time to resection, and identify factors associated with oncological transformation.

\section{Conclusions}

Our study found that GTR for pediatric patients suffering from epilepsy associated with low-grade neuronalglial tumors is strongly correlated with seizure-free outcome (84\%) and a lower recurrence rate. Further studies are needed to elucidate the most suitable time for surgery, to identify factors associated with oncological transformation, and to clarify the role of ECoG.

\section{References}

1. Bansal S, Kim AJ, Berg AT, Koh S, Laux LC, Nangia S, et al: Seizure outcomes in children following electrocorticog- 
raphy-guided single-stage surgical resection. Pediatr Neurol 71:35-42, 2017

2. Blümcke I, Aronica E, Becker A, Capper D, Coras R, Honavar M, et al: Low-grade epilepsy-associated neuroepithelial tumours - the 2016 WHO classification. Nat Rev Neurol 12:732-740, 2016

3. Englot DJ, Berger MS, Barbaro NM, Chang EF: Factors associated with seizure freedom in the surgical resection of glioneuronal tumors. Epilepsia 53:51-57, 2012

4. Englot DJ, Berger MS, Barbaro NM, Chang EF: Predictors of seizure freedom after resection of supratentorial low-grade gliomas. A review. J Neurosurg 115:240-244, 2011

5. Fallah A, Weil AG, Sur S, Miller I, Jayakar P, Morrison G, et al: Epilepsy surgery related to pediatric brain tumors: Miami Children's Hospital experience. J Neurosurg Pediatr 16:675-680, 2015

6. Faramand AM, Barnes N, Harrison S, Gunny R, Jacques T, Tahir MZ, et al: Seizure and cognitive outcomes after resection of glioneuronal tumors in children. Epilepsia 59:170 178, 2018

7. Fisher RS, Acevedo C, Arzimanoglou A, Bogacz A, Cross $\mathrm{JH}$, Elger CE, et al: ILAE official report: a practical clinical definition of epilepsy. Epilepsia 55:475-482, 2014

8. Giulioni M, Galassi E, Zucchelli M, Volpi L: Seizure outcome of lesionectomy in glioneuronal tumors associated with epilepsy in children. J Neurosurg 102 (3 Suppl):288-293, 2005

9. Giulioni M, Marucci G, Pelliccia V, Gozzo F, Barba C, Didato G, et al: Epilepsy surgery of "low grade epilepsy associated neuroepithelial tumors": a retrospective nationwide Italian study. Epilepsia 58:1832-1841, 2017

10. Guerrini R, Parrini E: Neuronal migration disorders. Neurobiol Dis 38:154-166, 2010

11. Huynh-Le MP, Walker AJ, Burger PC, Jallo GI, Cohen KJ, Wharam MD, et al: Management of pediatric intracranial low-grade gliomas: long-term follow-up after radiation therapy. Childs Nerv Syst 32:1425-1430, 2016

12. Ogiwara H, Nordli DR Jr, DiPatri AJ Jr, Alden TD, Bowman RM, Tomita T: Pediatric epileptogenic gangliogliomas: seizure outcome and surgical results. J Neurosurg Pediatr 5:271-276, 2010

13. Pelliccia V, Deleo F, Gozzo F, Sartori I, Mai R, Cossu M, et al: Early and late epilepsy surgery in focal epilepsies associated with long-term epilepsy-associated tumors. J Neurosurg 127:1147-1152, 2017

14. Ramantani G, Kadish NE, Anastasopoulos C, Brandt A, Wagner K, Strobl K, et al: Epilepsy surgery for glioneuronal tumors in childhood: avoid loss of time. Neurosurgery 74:648-657, 2014

15. Sarkis RA, McGinnis S, Rushia SN, Park S, Ansari EE, Willment KC: Growing older with drug-resistant epilepsy: cognitive and psychosocial outcomes. J Neurol 265:1059-1064, 2018

16. So EL: Integration of EEG, MRI, and SPECT in localizing the seizure focus for epilepsy surgery. Epilepsia 41 (Suppl 3):S48-S54, 2000
17. Southwell DG, Garcia PA, Berger MS, Barbaro NM, Chang EF: Long-term seizure control outcomes after resection of gangliogliomas. Neurosurgery 70:1406-1414, 2012

18. Tomita T, Volk JM, Shen W, Pundy T: Glioneuronal tumors of cerebral hemisphere in children: correlation of surgical resection with seizure outcomes and tumor recurrences. Childs Nerv Syst 32:1839-1848, 2016

19. Vecht C, Royer-Perron L, Houillier C, Huberfeld G: Seizures and anticonvulsants in brain tumours: frequency, mechanisms and anti-epileptic management. Curr Pharm Des 23:6464-6487, 2017

20. Vendrame M, Alexopoulos AV, Boyer K, Gregas M, Haut J, Lineweaver $\mathrm{T}$, et al: Longer duration of epilepsy and earlier age at epilepsy onset correlate with impaired cognitive development in infancy. Epilepsy Behav 16:431-435, 2009

21. Wick W, Menn O, Meisner C, Steinbach J, Hermisson M, Tatagiba M, et al: Pharmacotherapy of epileptic seizures in glioma patients: who, when, why and how long? Onkologie 28:391-396, 2005

22. Yao PS, Zheng SF, Wang F, Kang DZ, Lin YX: Surgery guided with intraoperative electrocorticography in patients with low-grade glioma and refractory seizures. J Neurosurg 128:840-845, 2018

\section{Disclosures}

Dr. Curry is a consultant for Medtronic, Inc., and Monteris, Inc.

\section{Author Contributions}

Conception and design: Aldave, Weiner, Yoshor. Acquisition of data: Mohan, Lee. Analysis and interpretation of data: Aldave, Mohan, Weiner. Drafting the article: Aldave, Mohan, Weiner. Critically revising the article: Aldave, Weiner, Mohila, Adesina, Chintagumpala, Curry, Jea, Lam, Whitehead, Dauser, Yoshor. Reviewed submitted version of manuscript: Aldave, Weiner, Mohila, Adesina, Chintagumpala, Curry, Jea, Lam, Whitehead, Dauser, Yoshor. Approved the final version of the manuscript on behalf of all authors: Aldave. Statistical analysis: Aldave, Mohan. Administrative/technical/material support: Aldave, Mohila. Study supervision: Aldave, Yoshor.

\section{Supplemental Information}

\section{Previous Presentations}

Part of this work was presented at the last AANS pediatric neurosurgery meeting, in Houston, TX, November 2017.

\section{Correspondence}

Guillermo Aldave: Texas Children's Hospital, Baylor College of Medicine, Houston, TX. gxaldave@texaschildrens.org. 